apuntesuniversitarios.upeu.edu.pe

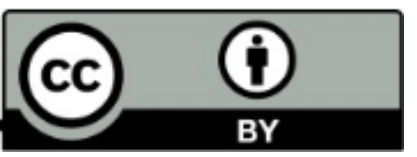

Apuntes Universitarios, 2020: 10(1), Enero-Marzo

ISSN: 2304-0335 DOI: https://doi.org/10.17162/au.v10i1.423

\title{
Mapas mentales armónicos (mma) como estrategia metacognitiva para la comprensión lectora en estudiantes universitarios
}

\section{Harmonic Mind Maps (MMA) as a metacognitive strategy for reading comprehension in university students}

Pedro Félix Novoa Castillo ${ }^{1}{ }^{\mathrm{a}}$ Luis Alberto Núñez Lira ${ }^{2}$ Flor de María Sánchez Aguirre, ${ }^{3}$ Florencio Flores Ccanto, ${ }^{4}$ Jorge Rafael Díaz Dumont ${ }^{5}$, Yrene Cecilia Uribe Hernandez
Universidad Nacional Mayor de San $\operatorname{Marcos}^{12}$
Universidad César Vallejo ${ }^{3}$
Universidad Nacional De Educación Enrique Guzmán y Valle ${ }^{4}$ Universidad
Nacional Autónoma de Tayacaja "Daniel Hernández Morillo",
Universidad Nacional de Cañete, Lima, Perú ${ }^{6}$
Orcid ID: https://orcid.org/0000-0003-2186-74581
D Orcid ID: https://orcid.org/0000-0003-3542-9117²
D Orcid ID: https://orcid.org/0000-0001-6416-6817 3
Orcid ID: https://orcid.org/0000-0001-5600-9854
Orcid ID: https://orcid.org/0000-0003-0921-338X
Orcid ID:https://orcid.org/0000-0001-5893-92626

Recibido: 26 de julio de 2019

Aceptado: 18 de octubre 2019

\section{Resumen}

La presente investigación se propuso determinar la influencia de los Mapas Mentales Armónicos (MMA) como estrategia metacognitiva en la comprensión lectora en estudiantes universitarios. Para ello se trabajó con 310 estudiantes de pregrado de la Facultad de Educación de la Universidad Nacional Mayor de San Marcos (UNMSM) durante el 2do semestre del 2019. La metodología estuvo enmarcada dentro del enfoque cuantitativo y se siguió el método hipotético deductivo. Por ello, su diseño fue el cuasiexperimental. El recojo de información se realizó mediante el Test CLUni 2015. Los resultados arrojaron que el empleo de los MMA como estrategia metacognitiva

${ }^{\text {a }}$ Correspondencia al autor

E-mail: pedro.felix.novoa.castillo@gmail.com 
incide de manera positiva y significativa en la mejora de la comprensión lectora de estudiantes universitarios.

Palabras-clave: Mapa Mental Armónico, TIC, Comprensión lectora, Estrategia metacognitiva, Metacognición.

\begin{abstract}
This research aimed to determine the influence of Harmonic Mind Maps (HMM) as a metacognitive strategy in reading comprehension in university students. For this, 310 undergraduate students from the Faculty of Education of the National University of San Marcos (UNMSM in spanish) were worked during the 2nd semester of 2019. The methodology was framed within the quantitative approach and the deductive hypothetical method was followed. Therefore, its design was quasi-experimental. The information was collected through the 2015 CLUni Test. The results showed that the use of MMA as a metacognitive strategy has a positive and significant impact on improving the reading comprehension of university students.
\end{abstract}

Keywords: Harmonic Mental Map, ICT, Reading Comprehension, Metacognitive Strategy, Metacognition.

\title{
Introducción
}

Se concibe la lectura como un procesamiento complejo, no natural y aprendido (León-Islas, López y Tamay, 2019), que se ejercita de manera diversa y desigual, condicionada tanto a factores físicos (visión, cansancio) como psicológicos (motivación, hábito); por lo que se hace necesario considerar alguna estrategia a fin de asegurar la eficacia de dicho proceso.

Lamentablemente, son pocas las estrategias que los universitarios aplican en sus ejercicios académicos, y las tradicionales como releer y eventualmente efectuar apuntes (Gallardo y López, 2019) las realiza un lector bisoño o negligente. Por otro lado, el lector experto suele orientarse al empleo de una estrategia metacognitiva que garantice la comprensión del texto durante el proceso. Asimismo, para que las estrategias metacognitivas sean efectivas, el profesor no debe esperar que se den espontáneamente, se tienen que enseñar (Campos, 2000). Y al estar a cargo del profesor, este debe planificar la clase eligiendo determinadas técnicas que sean lo más idóneas posibles.

Por ello, la presente investigación exhibe el empleo de los Mapas Mentales Armónicos (MMA) como una estrategia metacognitiva pertinente. Este tipo de organizador visual de información permite mapear paralelamente la lectura y el proceso de comprensión de la misma (Díaz y Hernández, 2002). Entendiendo que en la actualidad la búsqueda de optimizar tiempo y calidad de estudio es un imperativo ineludible (Perkins, 1992), se opta por una estrategia 
metacognitiva que permita solucionar problemas de manera efectiva, autodirigiendo su propio aprendizaje.

Por su parte, los MMA permiten cumplir todas las fases de la metacognición (Alcas, Alarcón, Gonzáles y Rodríguez, 2019) como planificar la lectura y el proceso de comprensión de la lectura; monitorear la lectura y regular la fluidez de la comprensión, y finalmente evaluar lo leído y la comprensión de lo leído simultáneamente; puesto que el lector experto, por lo general apela a la metacognición para auto regular los procesos realizados en la búsqueda de la comprensión íntegra de un texto (Griffith y Ruan, 2006).

\section{Problemática}

La realidad problemática de este trabajo se centra en las dificultades y poca comprensión lectora que manifiestan los estudiantes universitarios. Como precedente directo se encuentran los bajos resultados de las últimas pruebas PISA a los estudiantes de nivel secundario, los cuales podrían engrosar las filas de universitarios que aún presentan problemas en la comprensión lectora. Por este motivo, se decidió aplicar como instrumento de medición de la comprensión lectora, una prueba que se base en los criterios PISA, elaborada explícitamente para estudiantes de nivel superior, como el Test CLUni creado por Felipe, Barrios y Gonzáles (Málaga, 2015). El instrumento elaborado está conformado por seis lecturas con cuatro preguntas cada una, sumando en total veinticuatro ítems. Todos de alternativa múltiple y distribuida de la siguiente manera: 13 referidas a Comprensión superficial (Acceso y obtención de información); 9 a Comprensión profunda (Integración e interpretación); y 2 a Comprensión crítica (Reflexión y valoración). Estos son concordantes a la tipología de Werlich (1976), empleada también por PISA (2009 y 2012).

\section{Comprensión lectora}

Se entiende la comprensión lectora como el proceso activo entre el lector y un texto en la construcción de un sentido interpretativo, donde el leyente aprovecha sus conocimientos previos y los relaciona significativamente con el nuevo conocimiento que adquiere al leer (Antich, 1986; Solé, 1997; Snow, 2002). Por su parte, Sánchez y García-Rodicio (2014) establecen que la comprensión lectora puede ser de tres tipos: 1. Superficial, cuando el lector comprende lo que lee y ha sido capaz de seleccionar y organizar el contenido del texto, relacionándolos con sus saberes 
previos; 2. Profunda, cuando el lector comprende además de lo textual, el contexto situacional de la lectura, pues ha logrado integrar sus conocimientos previos con los nuevos conocimientos que ha adquirido al leer; y 3. Crítica, cuando el lector comprende lo leído de manera reflexiva, ya que ha conseguido valorar la consistencia y validez de lo leído y logra criticar y discriminar las inconsistencias del mismo, aportando al contenido del texto leído los conocimientos con los que ya cuenta. Estos tres tipos coinciden respectivamente con los aspectos propuestos por PISA, 2012 (como se citó en Felipe y Barrios, 2017): acceder y obtener, integrar e interpretar, y reflexionar y valorar.

Por otro lado, es importante resaltar que la mayoría de universidades en Latinoamérica cuenta con evaluaciones de comprensión lectora incluidas en sus exámenes de admisión, que posteriormente no son valoradas a fin de corroborar la competencia lectora. Solo un reducido grupo de investigaciones formulan alguna técnica, método o propuesta para mejorar la comprensión lectora en estudiantes universitarios (González, 1998; Novoa, 2012; Pizarro, 2008; Novoa et al, 2018; Novoa, 2019; Alcas et al, 2019). Es por ello que se decidió aportar, en la resolución de esta problemática, una propuesta metacognitiva a través de Mapas Mentales Armónicos, que son organizadores gráficos de potente significancia mental diseñados para esta investigación mediante el software miMind - Easy Mind Mapping; un programa disponible tanto para PC de escritorio y como aplicativo de dispositivos móviles (es de acceso libre).

\section{Los Mapas Mentales Armónicos (MMA) como estrategia metacognitiva}

Se eligieron los Mapas Mentales Armónicos (MMA), una variante de los mapas convencionales de Buzan propuesta en trabajos previos (Novoa et al, 2018; Novoa, 2019) como estrategia metacognitiva; entendiendo a los MMA como organizadores mejorados de información al reemplazar lo abigarrado (del original) por lo armónico (asegurado por uno de los diseños simétricos del software miMind).

Figura 1. Diseño simétrico del MMA 


\section{miMind}

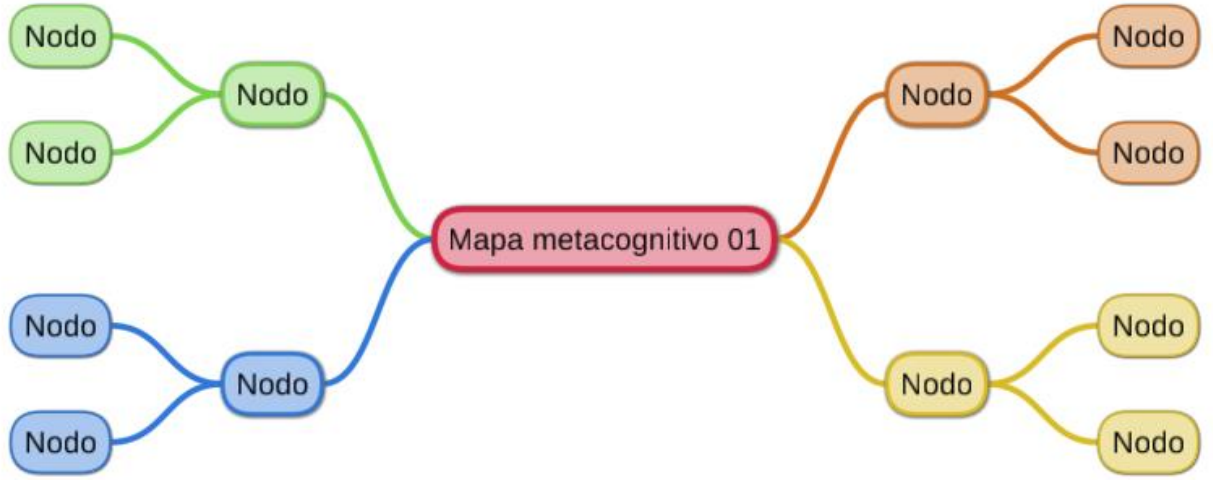

Fuente: Elaboración propia a través del miMind

Los MMA se adaptan muy bien como estrategia metacognitiva, asumiendo la metacognición como el proceso mental donde el ser humano tiene control de su propia cognición (Barraza, 2019). Esta suerte de metaconciencia ha sido aplicada a los MMA, siguiendo los procesos metacognitivos de Monereo (2000) quien menciona que cualquier estrategia metacognitiva debe tener corresponder a una planificación, control y autoevaluación de lo que se va comprendiendo por parte del propio estudiante. Bajo estas premisas la comprensión lectora de los 6 textos del Test de CLUni (2015) fueron mediadas por los MMA que planificaron, controlaron y autoevaluaron la comprensión organizando la siguiente información: Tema principal y temas secundarios, imagen alusiva a la lectura, clase de texto, forma del texto, posibles respuestas y algún otro dato que el lector crea pertinente.

Figura 2. MMA de la primera lectura 


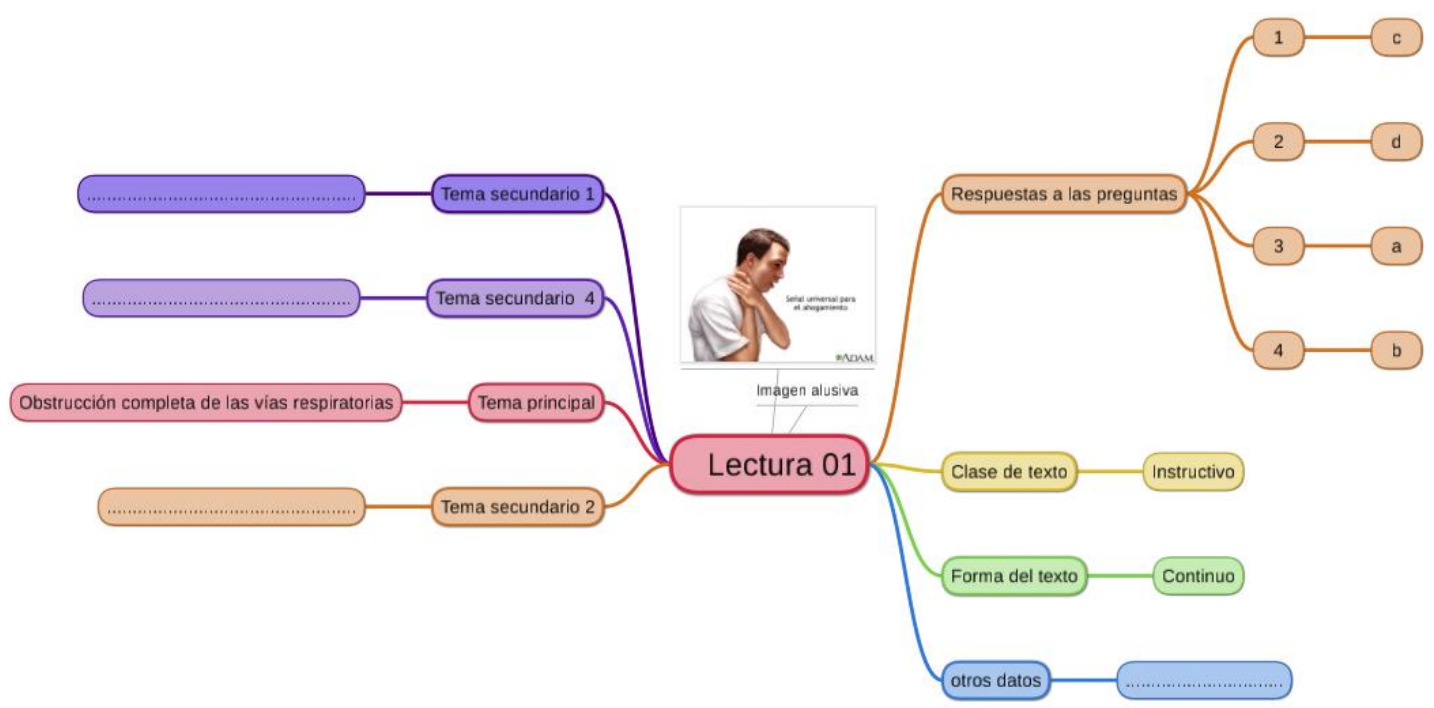

Fuente: Elaboración propia a través del miMind

\section{Metodología}

Esta investigación estuvo encuadrada desde el paradigma neopositivista y el enfoque cuantitativo, entendiéndola como la apelación a la ciencia (informática y estadística) como corroborador y predictor de sus resultados. El método fue el hipotético deductivo, pues se partió de la hipótesis basada en la teoría y trabajos previos que indicaban la incidencia de estrategias metacognitivas en procesos mentales como la comprensión lectora. El diseño del estudio es de tipo experimental en su modalidad cuasi experimental. Asimismo, se aplicó un corte temporal transversal, ya que la evaluación de la variable dependiente (comprensión lectora) se midió en dos momentos puntuales (pre y postest). Cabe destacar el lapso de tiempo de la experimentación: dos semanas.

\section{Población y muestra}

El universo poblacional estuvo conformado por 1600 estudiantes de ambos sexos, matriculados en el año 2019-II de la Facultad de Educación de la UNMSM. La muestra fue de 310 participantes obtenida según la siguiente fórmula:

Figura 3. Fórmula de cálculo de la muestra 
Margen: $5 \%$

Nivel de confianza: $95 \%$

Poblacion: 1600

Tamaño de muestra: $\underline{310}$

$$
\begin{aligned}
& \text { Ecuacion Estadistica para Proporciones } \\
& \text { poblacionales } \\
& \mathrm{n}=\text { Tamaño de la muestra } \\
& \mathrm{z}=\text { Nivel de confianza deseado } \\
& \mathrm{p}=\text { Proporcion de la poblacion con la caracteristica } \\
& \text { deseada (exito) } \\
& \mathrm{q}=\text { Proporcion de la poblacion sin la caracteristica } \\
& \text { deseada (fracaso) } \\
& \mathrm{e}=\text { Nivel de error dispuesto a cometer } \\
& \mathrm{N}=\text { Tamaño de la poblacion }
\end{aligned}
$$

Fuente: tomado de http://www.corporacionaem.com/tools/calc muestras.php

A fin de conformar la muestra anterior se optó por un muestreo probabilístico del tipo aleatorio simple, entendiéndolo como la técnica muestral que apela al azar, donde cualquier sujeto

\begin{tabular}{|c|c|c|c|}
\hline Dimensiones & Indicadores / Escala & Ítem & Niveles y rangos \\
\hline $\begin{array}{l}\text { Comprensión } \\
\text { Superficial }\end{array}$ & $\begin{array}{l}\text { Accede y obtiene información del texto, } \\
\text { relacionándolos con sus saberes previos. } \\
\text { Escala }(0=\text { incorrecto/ } 1=\text { Correcto) }\end{array}$ & $\begin{array}{l}3,8,9,11,12,13,14,15,16 \\
19,21,22,23\end{array}$ & \\
\hline $\begin{array}{l}\text { Comprensión } \\
\text { Profunda }\end{array}$ & $\begin{array}{l}\text { Interpreta lo leído, integrando sus } \\
\text { saberes previos con los nuevos } \\
\text { conocimientos. } \\
\text { Escala ( } 0=\text { incorrecto/ } 1=\text { Correcto) }\end{array}$ & $1,2,4,5,6,7,10,17,18$ & Baja (0-7) \\
\hline
\end{tabular}
de la población tiene la posibilidad de ser considerado en la muestra (Malhotra, 2008). Para asegurar esto, se hizo uso de herramientas de diversas escuelas (Lengua, Literatura y comunicación, Educación física, Primaria, entre otras). Las cantidades representativas se tomaron por escuelas utilizando el utilitario de "Muestra de casos aleatorios" al 5\% del software SPSS. De la misma manera, se eligió a los grupos de control y experimental, quedando la muestra en 310 participante distribuida de la siguiente manera: Grupo control (160) y Grupo experimental (150).

\section{Operacionalización de la variable dependiente}

\section{Tabla 2}

Operacionalización de la variable Comprensión lectora 
Comprensión Crítica
Reflexiona y valora la consistencia y 20,24

validez de lo leído como también la crítica.

Escala ( $0=$ incorrecto/ $1=$ Correcto)
Media (8-15)

Alta (16-24)

\section{Resultados}

\section{Resultados descriptivos}

Luego de dos semanas de aplicación estratégica de los MMA, se aplicó la misma para la comprensión lectora (Test CLUni, 2015), teniendo los siguientes resultados comparativos entre el grupo control y el experimental:

Tabla 3

Comprensión lectora por grupos y test

\begin{tabular}{llcccc}
\cline { 3 - 5 } Comprensión lectora & pre control & pre experimental & pos control & $\begin{array}{c}\text { pos } \\
\text { experimental }\end{array}$ \\
\cline { 3 - 6 } & Baja & 58 & 40 & 15 & 14 \\
& Media & 92 & 93 & 102 & 39 \\
& Superior & 10 & 17 & 43 & 97 \\
Total & & 160 & 150 & 160 & 150 \\
\hline
\end{tabular}

Figura 4. Comprensión lectora por grupos y test 


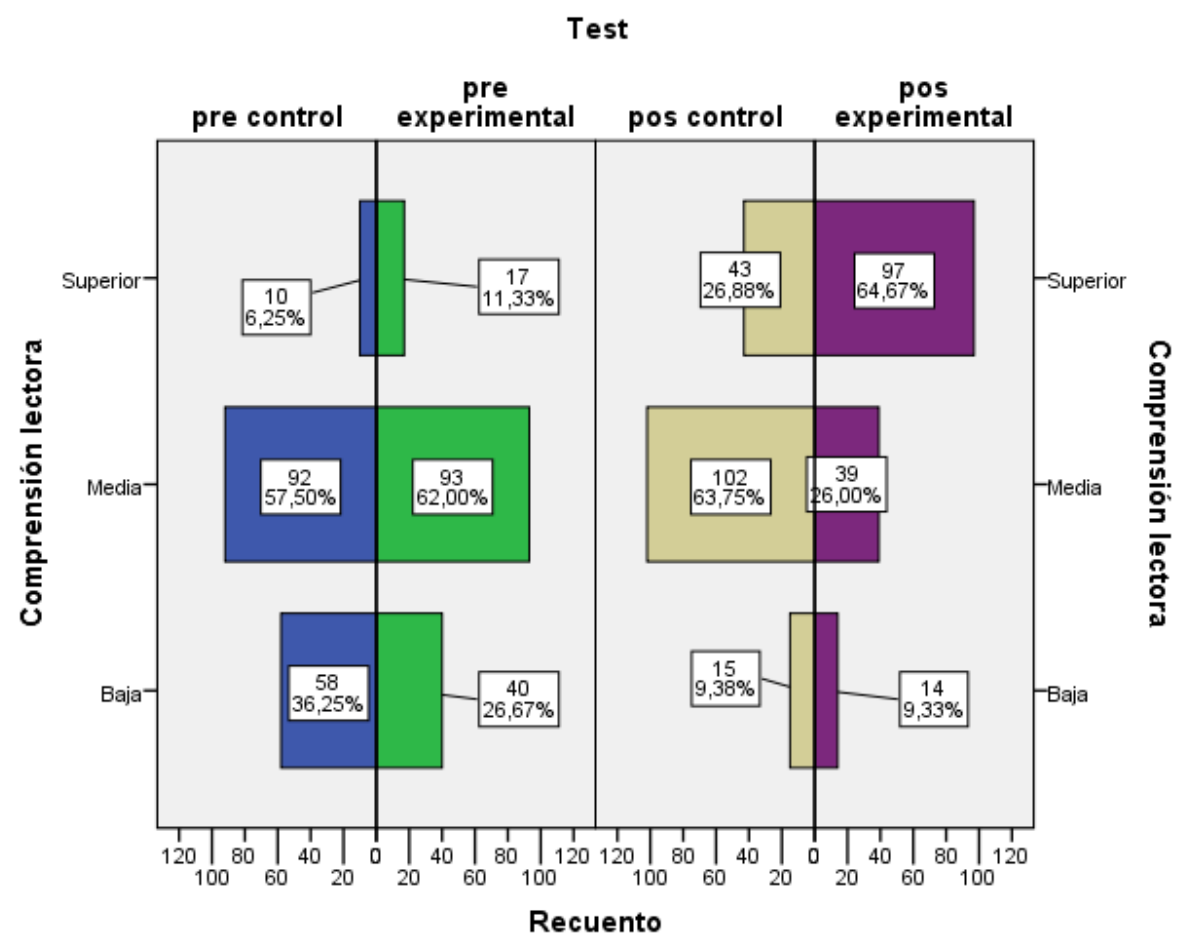

Fuente: Elaboración propia a través de los resultados SPSS

Según se observa en la Tabla 3 y la Figura 4, en el pretest, tanto el grupo control como el grupo experimental no manifiestan diferencias marcadas, predominando en ambos una comprensión media. Al contrario, en el postest se observa una evidente diferencia a favor del grupo experimental en el nivel superior de comprensión. Asimismo, se interpreta que se habría producido un desplazamiento cualitativo del nivel medio al superior en el grupo experimental por el efecto del uso de los MMA.

Figura 5. Comprensión superficial por grupos y test 


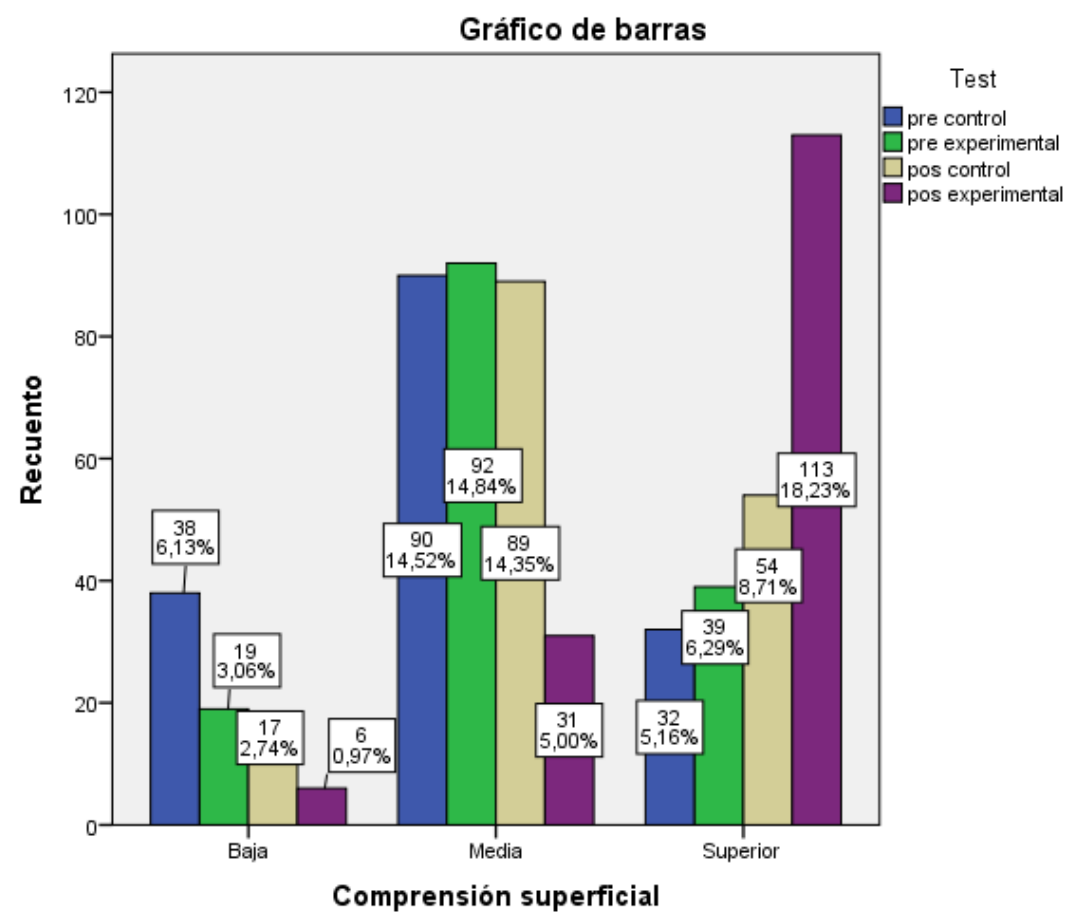

Fuente: Elaboración propia a través de los resultados SPSS

Por otro lado, se evidencia que tanto el grupo control y el grupo experimental mejoraron sus niveles de comprensión, pues ambos abandonaron el nivel bajo; asimismo, en el nivel medio se observa que el grupo de control se mantuvo en su nivel, mientras que el experimental advirtió una baja. Esto se interpreta con el análisis del nivel superior, donde se observa que, de los dos grupos, es el experimental quien ha obtenido un gran salto cualitativo.

Figura 6. Comprensión profunda por grupos y test 


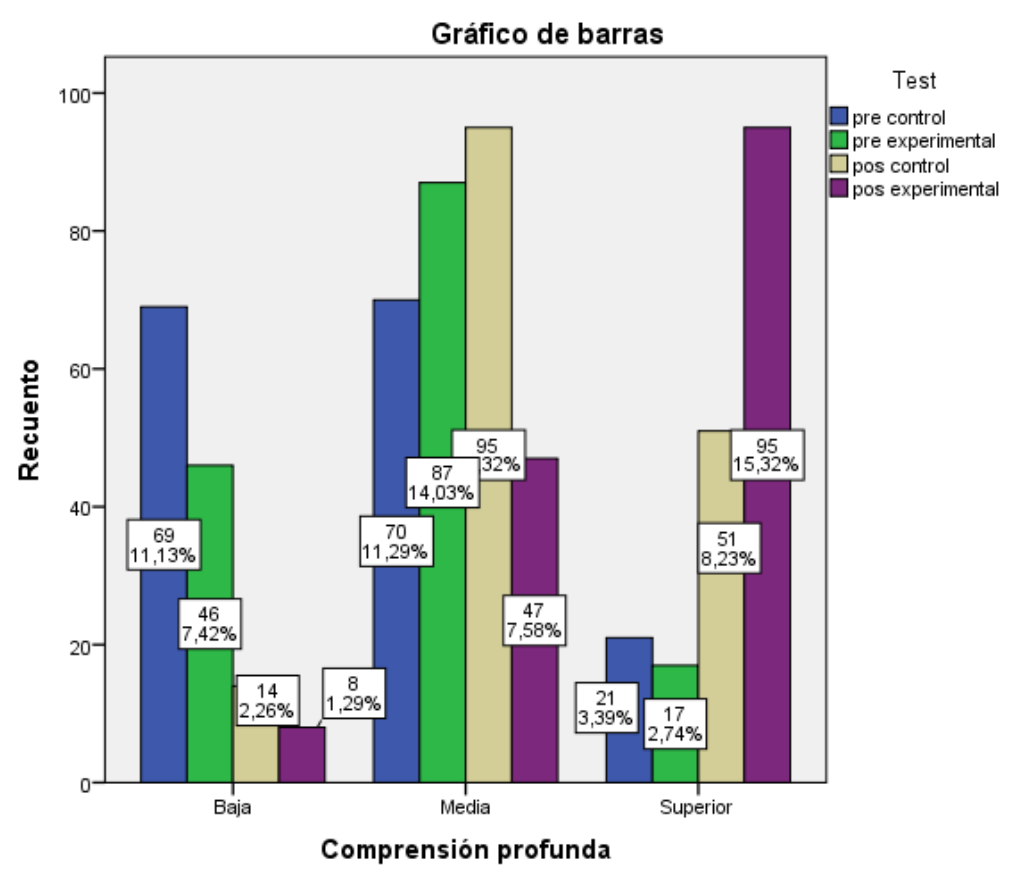

Fuente: Elaboración propia a través de los resultados SPSS

Por otra parte, se observa que, tanto el grupo control y el grupo experimental mejoraron sus niveles de comprensión, pues ambos abandonaron el nivel bajo, sobre todo el grupo control; asimismo, en el nivel medio se observa que el grupo de control ascendió, mientras que el experimental bajó. Esto se comprende mejor luego del análisis del nivel superior, donde se muestra que el grupo experimental ha obtenido un desplazamiento cualitativo, un ascenso con algunos puntos por debajo que la anterior dimensión, muy probablemente porque la comprensión profunda es más difícil que la superficial.

Figura 7. Comprensión crítica por grupos y test 


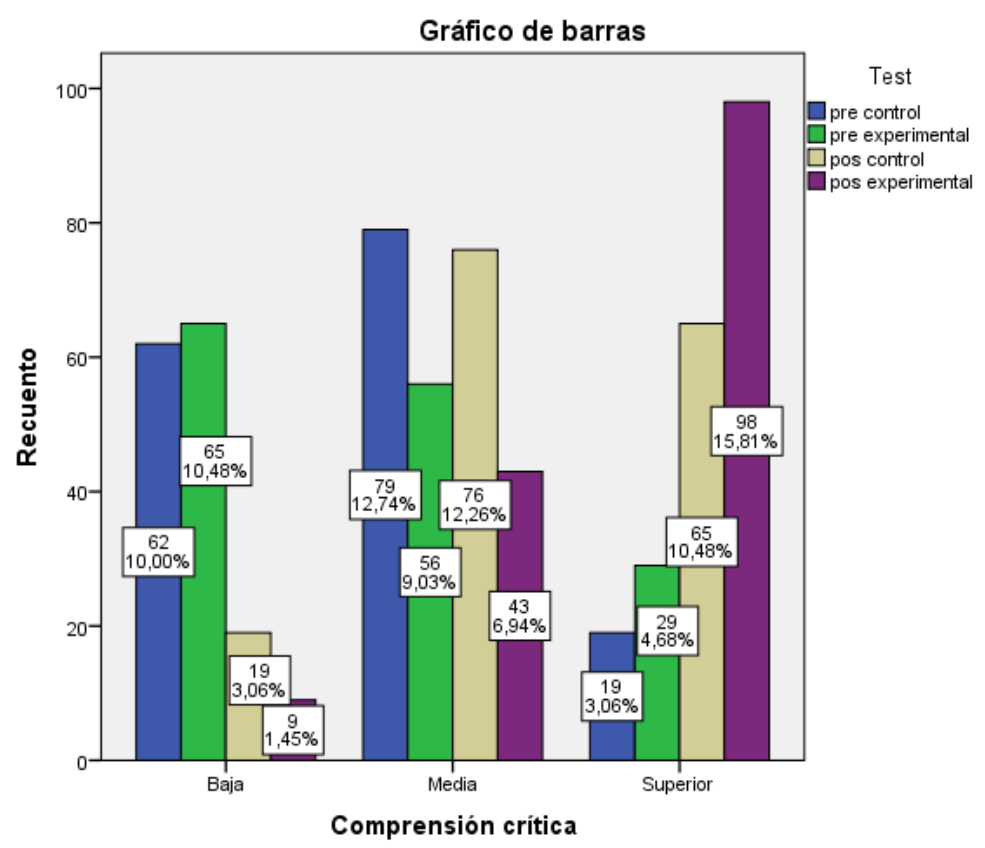

Fuente: Elaboración propia a través de los resultados SPSS

En esta última figura se demuestra que tanto el grupo control y el grupo experimental mejoraron sus niveles de comprensión, pues ambos abandonaron el nivel bajo, sobre todo el experimental; asimismo, en el nivel medio se observa que el grupo de control descendió al igual que el experimental; esto se interpreta mejor luego del análisis del nivel superior, donde se muestra el ascenso de ambos grupos, unos puntos por debajo que la dimensión profunda, probablemente porque la comprensión profunda es más compleja que la crítica, así como en el número mayor de preguntas que la crítica.

\section{Resultados inferenciales}

El nivel de significancia es $\alpha=0.05$, correspondiente a una confiabilidad del $95 \%$, debido a que se emplearon datos no paramétricos, aplicándose la Prueba U de Mann-Whitney.

Rechazar $\mathrm{H}_{0}$ cuando el "p" valor sea menor que $\alpha$.

No rechazar $\mathrm{H}_{0}$ cuando el " $\mathrm{p}$ " valor sea mayor que $\alpha$.

\section{Prueba de hipótesis general}


H0: La aplicación de los MMA como estrategia metacognitiva no incide de manera positiva en la comprensión lectora.

Hi: La aplicación de los MMA como estrategia metacognitiva incide de manera positiva en la comprensión lectora.

\section{Prueba de hipótesis específica}

H0: La aplicación de los MMA como estrategia metacognitiva no incide de manera positiva en la comprensión superficial, profunda y crítica.

Hi: La aplicación de los MMA como estrategia metacognitiva incide de manera positiva en la comprensión superficial, profunda y crítica.

Tabla 4

Rangos de la Comprensión lectora y sus dimensiones en el pre test

\begin{tabular}{ccccc}
\hline & Test & N & Rango promedio & Suma de rangos \\
\hline Comprensión lectora & pre control & 160 & 146,33 & 23413,00 \\
& pre experimental & 150 & 165,28 & 24792,00 \\
Comprensión superficial & pre control & 160 & 145,42 & 23267,00 \\
& pre experimental & 150 & 166,25 & 24938,00 \\
Comprensión profunda & pre control & 160 & 148,15 & 23703,50 \\
& pre experimental & 150 & 163,34 & 24501,50 \\
Comprensión crítica & pre control & 160 & 155,10 & 24816,50 \\
& pre experimental & 150 & 155,92 & 23388,50 \\
& Comprensión & Comprensión & Comprensión & Comprensión \\
& lectora & superficial & profunda & crítica \\
U de Mann-Whitney & 10533,000 & 10387,000 & 10823,500 & 11936,500 \\
Z & $-2,140$ & $-2,317$ & $-1,650$ &,- 088 \\
Sig. asintótica & & & &, 930 \\
(bilateral) &, 032 &, 021 &, 099 & \\
\hline
\end{tabular}

Tabla 5 
Prueba de U de Mann-Whitney de la Comprensión lectora y sus dimensiones en el pos test

\begin{tabular}{|c|c|c|c|c|}
\hline & Test & $\mathbf{N}$ & Rango promedio & Suma de rangos \\
\hline \multirow[t]{2}{*}{ Comprensión lectora } & pos control & 160 & 129,79 & 20766,50 \\
\hline & pos experimental & 150 & 182,92 & 27438,50 \\
\hline Comprensión & pos control & 160 & 124,33 & 19893,50 \\
\hline superficial & pos experimental & 150 & 188,74 & 28311,50 \\
\hline \multirow[t]{2}{*}{ Comprensión profunda } & pos control & 160 & 132,23 & 21156,00 \\
\hline & pos experimental & 150 & 180,33 & 27049,00 \\
\hline \multirow[t]{4}{*}{ Comprensión crítica } & pos control & 160 & 136,55 & 21848,50 \\
\hline & pos experimental & 150 & 175,71 & 26356,50 \\
\hline & Comprensión & Comprensió & Comprensión & Comprensión \\
\hline & lectora & n superficial & profunda & crítica \\
\hline U de Mann-Whitney & 7886,500 & 7013,500 & 8276,000 & 8968,500 \\
\hline $\mathbf{Z}$ & $-5,785$ & $-7,135$ & $-5,282$ & $-4,305$ \\
\hline $\begin{array}{c}\text { Sig. asintótica } \\
\text { (bilateral) }\end{array}$ & ,000 &, 000 &, 000 &, 000 \\
\hline
\end{tabular}

Se observa en la tabla 4 que en el pretest de Comprensión lectora los rangos del grupo de control y el grupo experimental no son tan diferenciados. Asimismo, tanto el valor p. de significancia (mayor que $\alpha=, 05$ ) y el valor $Z$ (mayor que el límite crítico -1,96) corroboran que no existen diferencias significativas entre ambos grupos. Sin embargo, en el la tabla 5 del postest, sí hay una marcada diferencia en los rangos. Además, se aprecia que el p. de significancia (mayor que $\alpha=, 05)$ y el valor $Z$ (menor que el límite crítico -1,96) corroboran la presencia de diferencias significativas entre ambos grupos. Por tanto, se rechaza la hipótesis nula y se concluye que la aplicación de los MMA como estrategia metacognitiva incide de manera positiva en la comprensión lectora.

Por su parte, se muestra en la tabla 4 que en el pretest de Comprensión superficial, Comprensión profunda y Comprensión crítica, los rangos del grupo de control y el grupo experimental no presentan diferenciación marcada, y tanto el valor p. de significancia (mayor que $\alpha=, 05)$ y el valor $Z$ (mayor que el límite crítico -1,96) corroboran que no existen diferencias significativas entre ambos grupos. Sin embargo, en el la tabla 5 del postest, sí se presentan diferencias marcadas en los rangos. Además, se observa que el p. de significancia (mayor que $\alpha=$ ,05) y el valor Z (menor que el límite crítico -1,96) confirman diferencias significativas entre ambos grupos, favoreciendo al grupo experimental. Por tanto, se rechaza la hipótesis nula y se 
concluye que la aplicación de los MMA como estrategia metacognitiva incide de manera positiva en la Comprensión superficial, Comprensión profunda y Comprensión crítica.

\section{Discusión y conclusiones}

El presente estudio llega a la conclusión de que la aplicación de los MMA como estrategia metacognitiva incide de manera positiva en la Comprensión lectora. Esto coincide con González (1998); Pizarro (2008); Novoa et al (2018); y Novoa (2019); a pesar que se emplearon métodos, técnicas o estrategias diferentes, así como instrumentos distintos para la evaluación de los resultados. Se remarca también en el presente trabajo las ligeras ventajas en los resultados, muy probablemente por el nivel metacognitivo de auto reflexión que implica estar más consciente cuando se está ejerciendo un proceso mental complejo como es la comprensión lectora.

Por otro lado, se concluye que la aplicación de los MMA como estrategia metacognitiva incide de manera positiva en la Comprensión superficial, Comprensión profunda y Comprensión crítica. Esto se ubica en contraste con lo hallado por González (1998), Pizarro (2008), Novoa (2012) quienes a pesar de haber logrado resultados significativos remarcan el aún bajo nivel predominante de la comprensión. Asimismo, los hallazgos de esta investigación representan un avance cualitativo con lo hallado por Novoa et al (2018), Alcas et al (2019) y Novoa (2019) entendiendo que además de los niveles superiores de comprensión conseguidos, el sesgo metacognitivo agrega significatividad en los estudiantes universitarios al momento de comprender diversos tipos de textos.

Dentro de los resultados no esperados, se observa un nivel de comprensión mayor cuando el alumno pasa la metacognición de lo explícito a lo implícito, es decir, que se autorregula casi de manera inconsciente. Esto no es malo, pero ameritaría una investigación adicional sobre este deslizamiento cognitivo hacia lo espontáneo y natural.

\section{Recomendaciones}

- El campo de estudio sobre estrategias cognitivas y su relación con las TIC requiere mayor profundización en su análisis y aplicación, ya que en los países mejor posicionados en comprensión se suele vincular estos dos aspectos en gran manera. 
- En cuanto al instrumento para medir la comprensión, se sugiere elaborar uno destinado a la realidad de cada país, o por lo menos hallar una adaptación latinoamericana para el nivel universitario. Actualmente se viene realizando precisamente una adaptación del Test CLUni para Latinoamérica.

- Finalmente, es importante renovar las pruebas de comprensión, así como las técnicas y estrategias, sin prescindir de las exitosas prácticas convencionales como la lectura al aire libre, y las actividades de socialización de lecturas en grupo o en clubes de lectura.

\section{Referencias}

Aiken, L. (2003). Test psicológicos y evaluación. México: Pearson Educación.

Alcas, N., Alarcón, M. A., Alarcón, H. H., Gonzáles, R., \& Rodríguez, A. (2019). Estrategias metacognitivas y comprensión lectora en estudiantes universitarios.

Anastasi, A. (1986). Los tests psicológicos. Madrid: Aguilar.

Antich, L. R. (1986). Metodología de la Enseñanza de Lenguas Extranjeras. La Habana. Cuba: Editorial Pueblo y Educación; Vol. I: 23 - 32.

Barraza López, E. (2019). Habilidades metacognitivas y rendimiento académico en el área de matemática en los estudiantes del cuarto grado de nivel secundaria de la institución educativa "san pedro de Chorrillos"-Lima, 2017.

Bernal, M. I. (2012). Colombia 'se raja' en lecturabilidad de libros, revistas y periódicos frente a la Región. Diario La República, Colombia. 17 de abril 2012. Infografía del Centro Regional para el Fomento del Libro en América Latina y el Caribe (Cerlalc). Recuperado de https://www.larepublica.co/ocio/colombia-se-raja-en-lecturabilidad-de-librosrevistas-y-periodicos-frente-a-la-region-2007794, consultado el 31 de octubre de 2019.

Campos, Y. (2000). Estrategias de enseñanza aprendizaje. Estrategias didácticas apoyadas en Tecnología. Obtenido de la Universidad Autónoma Metropolitana: http://virtuami.izt.uam.mx/eportafolio/DocumentosApoyo/estrategiasenzaprendizaje.pdf

Delgadová, E., \& Gullerová, M. (2015). Comprensión lectora. Un estudio sobre la competencia lectora en el contexto universitario. Lenguajes y Textos, 41, 45-53.

Díaz, F., \& Hernández, G. (2002). Estrategias docentes para un aprendizaje significativo (Vol. 2). México: McGraw-Hill. 
Felipe, A. (2015). Competencia, estrategias y hábitos lectores de maestros en formación inicial (Tesis doctoral). Málaga: Universidad de Málaga.

Felipe, A. M. \& Barrios, E. E. (2017). Evaluación de la competencia lectora de futuros docentes. Investigaciones sobre lectura, (7), 7-21.

Gallardo, L. S. y López, M, E. (2019). Conocimiento y uso de estrategias de comprensión lectora en estudiantes de psicología. Revista Electrónica de Psicología Iztacala, 22(2), 20662086.

Gonzáles Moreyra, R. (1998). Nivel de comprensión lectora en los universitarios iniciales. (Tesis de maestría), Universidad Nacional Mayor de San Marcos, Lima, Perú.

González Álvarez, C. (2011). Didáctica de la Lengua en la Escuela Infantil. Granada: Grupo Editorial Universitario.

Griffith, P. L., \& Ruan, J. (2006). What is metacognition and what should be its role in literacy instruction?. In Metacognition in literacy learning (pp. 25-40). Routledge. Recuperado de https://www.taylorfrancis.com/books/e/9781410613301/chapters/10.4324/97814106133 $\underline{01-10}$

Hopper, C. (2016). Practicing College Learning Strategies ( $7^{\mathrm{a}}$ ed.). EEUU: Cengage Learning. https://dx.doi.org/10.24320/redie.2017.19.2.1125

León-Islas, E. E., López, M. M., \& Tamay, J. A. C. (2019). Comprensión lectora y medición de fluidez en universitarios de origen maya. CPU-e, Revista de Investigación Educativa, (28), 152-182.

Malhotra, N. (2008). Investigación de Mercados (5 $5^{\mathrm{a}}$ ed.). Naucalpan de Juárez, México: Pearson Educación de México

Martins, A. (2016) Pruebas PISA: ¿cuáles son los países que tienen la mejor educación del mundo? ¿Y cómo se ubica América Latina? BBC Mundo, 06 de diciembre de 2016. https://www.bbc.com/mundo/noticias-38211248, consultado el 31 de octubre de 2019.

Monereo, C. (2000). Estrategias de enseñanza y aprendizaje. Formación del profesorado y aplicación a la escuela. Lima: Editorial Perú.

Muñoz, P., Muñoz, Amparo, E., Rosero, R., y Villota, C. (2008) Enseñanza de la producción escrita del cuento en la básica primaria. Actualidades Pedagógicas, vol. I, núm. 52, pp. 149-158 en https://bit.ly/2PBftVY

Novoa Castillo, P. (2012) Uso de los Mapas Mentales como instrumento de enseñanza en la comprensión lectora de textos narrativos en los alumnos del I Ciclo de la Universidad de 
Ciencias y Humanidades (Tesis de maestría, Universidad Nacional de Educación Enrique Guzmán y Valle).

Novoa Castillo, P. F., Cancino Verde, F., Flores Sotelo, W. S., \& Nieto Gamboa, J. (2018). El Mapa Mental Armónico en la comprensión de textos narrativos en estudiantes universitarios. Propósitos y Representaciones, 6(2), 541-573 en http://dx.doi.org/10.20511/pyr2018.v6n2.243.

Organización para la Cooperación y el Desarrollo Económicos (OCDE) (2006). PISA 2006. Marco de evaluación. Conocimientos y habilidades en Ciencias, Matemáticas y Lectura. Santillana Educación. Recuperado de http://www.oecd.org/pisa/39732471.pdf

Perkins, D. (1992) La escuela Inteligente. Editorial Gedisa: Barcelona, España.

Piacente, T. y Tittarelli, A. (2006), "Comprensión y producción de textos en alumnos universitarios: la reformulación textual”, Orientación y sociedad, vol. 6, núm.1, pp. 99126 en https://bit.ly/2zu5XOR

Pizarro Cherre, F. (2008). Aplicación de los mapas mentales en la comprensión lectora en estudiantes del ciclo I de instituciones de educación superior. (Tesis de maestría). Universidad Nacional Mayor de San Marcos, Lima, Perú.

Ribas, R., \& Hofmann, S. (2013). Don de Lenguas. Barcelona: Círculo de Lectores.

Sánchez, M. \& García-Rodicio, H. (2014). Comprensión de textos. Conceptos básicos y avances en la investigación actual. Aula, 20, 83-103.

Snow, C. (2002). Reading for understanding. Toward a Research and Development Program in Reading Comprehension. Santa Mónica:RAND Corporation.

Solé, I. (1997). La lectura, un proceso estratégico. Revista Signos Nro.20. España, Madrid.

Werlich, E. (1976). A Text Grammar of English. Heidelberg: Quelle and Meyer. 